\title{
Saccades to targets in three-dimensional space: Dependence of saccadic latency on target location
}

\author{
H. HONDA and J. M. FINDLAY \\ University of Durham, Durham, England
}

\begin{abstract}
The latency of saccadic movements to targets appearing at various positions in three-dimensional visual space was measured in four experiments. The first experiment confirmed that latencies of saccades to visual targets are greater in the lower visual field and showed that the increase is not influenced by the vertical starting position of the eye in the orbit, nor by a time gap between the fixation offset and the target onset. A hypothesis that this visual field difference was caused by a link between downward saccades and convergence movements was tested by recording saccade latencies when the targets were in a different depth plane from that of the original fixation. We did not find any direct support for the vergence involvement hypothesis, although the lower/upper visual field effect was shown to decrease consistently in monocular viewing. It was also shown that saccades to targets positioned in a different depth plane have longer latencies. In a final experiment, the visual field effect was shown to depend on the egocentric rather than the gravitational vertical.
\end{abstract}

When a subject makes a saccadic eye movement to a newly appearing target in the visual field, the latency provides a measure of the programming time for the movement. Although saccade latencies have been investigated in a number of studies, most frequently these studies have been done with targets that appeared on a horizontal axis to the left or right of the fixation point in the same plane as that of the original fixation. A review of such studies (Findlay, 1983) suggested that for suprathreshold targets on the horizontal axis, the latency of target-elicited saccades is generally independent of the position of the target over the range $1^{\circ}-15^{\circ}$.

Systematic latency differences have been reported when the target is not on the horizontal axis, however. A consistent increase of about $20 \mathrm{msec}$ in latency has been found when saccades are elicited to targets in the lower visual field (Hackman, 1940; Heywood \& Churcher, 1980; Miles, 1936). In an earlier study by one of the present authors (Findlay \& Harris, 1984), a similar increased latency to targets in the lower visual field was also noted as an incidental finding, although it was not reported. Only one study (Miller, 1969) has not replicated this finding; in that study, a (nonsignificant) difference was reported, averaging $12 \mathrm{msec}$ in the opposite direction. The saccade latencies, however, were abnormally long. (Note-we have not been able to trace the report by Heywood and

The assistance of Matthew Wenban-Smith in Experiment 1 is gratefully acknowledged. Experiments 2-4 were carried out while $\mathrm{H}$. Honda was spending a period of sabbatical leave at Durham. The experiments were supported by SERC from GR/E/88165. H. Honda is currently in the Department of Psychology at Niigata University, Ikarashi, Niigata 950-21, Japan. Correspondence concerning this article, including requests for reprints, should be sent to J. M. Findlay, Department of Psychology, University of Durham, South Road, Durham, DH1 3LE, England.
Churcher, 1980, of a contrary finding in a paper by Cohen and Ross.)

Our first aim in the present study was to confirm the previously reported longer latencies of saccades to targets in the lower visual field. Our second aim was to examine some possible factors that might be expected to produce or influence the lower visual field effect. It seems unlikely that the effect occurs at the level of sensory processing, since, as reviewed by Previc (1990), manual reaction times show the opposite pattern, being generally slightly faster for targets in the lower visual field. We were interested in the possibility that the lower visual field effect might be caused by a link between downward saccades and vergence eye movements. This is suggested by the fact that under most normal circumstances of viewing, objects and surfaces in the lower visual field are closer to the observer than those in the upper visual field, because of the natural ecology of visual space. Thus, under normal viewing, targets in the lower visual field will generally be closer than the original fixation point. Certain consequences for vision seem likely to be associated with this fact; one example would be the tilt of the vertical horopter (Howard, 1982; Nakayama, 1977). A consequence involving eye movements is that downward saccades will frequently be co-programmed with a convergence movement. Enright (1984, 1989) and Collewijn, Erkelens, and Steinman (1988) have found that downward saccades have a small nonconjugate converging component even when saccades are made to targets vertically below and at the same distance as the fixation point. For these targets, no convergence is required, and the saccade is followed by a slow corrective divergence movement. Could the increased latency for downward saccades be accounted for by the association with convergence, possibly because extra programming time was required? This 
possibility was tested in Experiment 2. We compared the latencies of saccades made to targets in the upper and the lower visual fields, with some of the targets at the same, and some at different, distances from the observer.

Unexpectedly, in Experiment 2, a general increase in latency was found for all saccades made to targets in a depth plane different from that of the original fixation. In Experiment 3, we investigated whether or not this increase was dependent on the target's being viewed binocularly. Finally, in Experiment 4, we examined whether the up/down latency difference was related to the gravitational vertical or to the egocentric vertical.

\section{EXPERIMENT 1}

Experiment 1 was conducted to confirm the previously reported increase in saccade latencies for targets in the lower visual field and to examine whether this effect could be influenced by two factors. The factors were, first, the vertical position of gaze and, second, the temporal relationship between fixation point offset and target onset. Saslow (1967) showed that saccade latencies could be shortened considerably by introducing a time gap between the offset of the fixation point and the onset of the target. More recent studies have suggested that some saccades in the gap conditions may constitute a separate type of saccade, the express saccade (Fischer, Boch, \& Ramsperger, 1984; Fischer \& Ramsperger, 1984), although this distinction has been questioned (Wenban-Smith \& Findlay, 1991). Attentional factors are often invoked to account for the decrease in latency in the gap conditions, and we thus hoped to discover whether these attentional factors would interact with the up/down latency difference.

\section{Method}

Subjects. Five Durham University faculty and graduate students with normal or corrected vision served as subjects (including the author, J.F.); they were 21-46 years old.

Stimuli and Procedure. The stimuli for this experiment were presented on the screen of a microcomputer (BBC Model B). The screen could be positioned directly in front of the subject at a viewing distance of $72 \mathrm{~cm}$, or it could be moved vertically (with a corresponding slight increase in viewing distance) to positions that required the gaze to be elevated by $20^{\circ}$ or depressed by $20^{\circ}$. In all cases, the screen was mounted vertically so that the upper visual field and lower visual field targets were displayed at the same point on the raster scan, which was synchronized to the eye movement recording routines.

The subjects saw a fixation cross at the center of the screen, which was otherwise uniformly illuminated $\left(19 \mathrm{~cd} / \mathrm{m}^{2}\right)$. After a time (variable between 1.0 and $1.5 \mathrm{sec}$ ), the cross disappeared and was replaced by a target that consisted of a square with sides of $0.75^{\circ}$, uniformly illuminated at a level of $69 \mathrm{~cd} / \mathrm{m}^{2}$. The square could appear either $4.8^{\circ}$ above or $4.8^{\circ}$ below the fixation point (in the central position) with equal probability. There were three gap conditions: the target could appear at the same time as the fixation point offset, it could be delayed by $100 \mathrm{msec}$, or it could be delayed by $200 \mathrm{msec}$. Sixteen trials at each combination of target direction and gap condition were presented within each block of 96 trials. The subjects carried out two blocks at each gaze position, in a balanced order. Viewing was binocular.

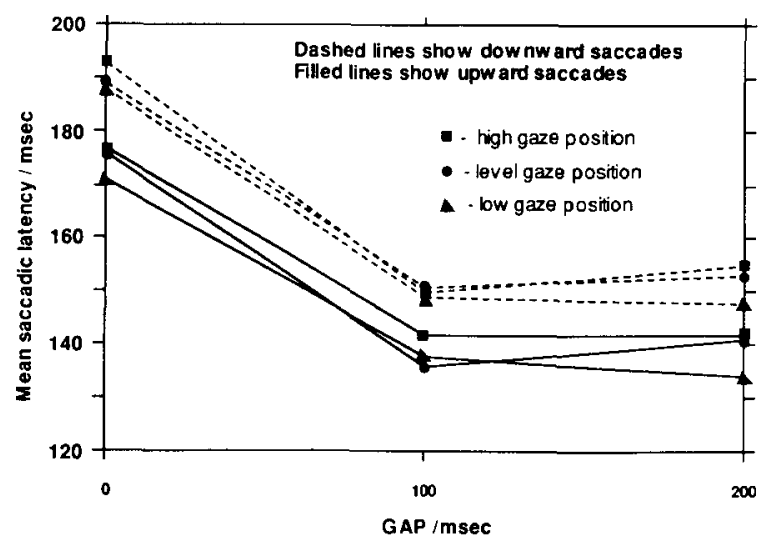

Figure 1. Saccade latencies for different viewing positions and gap durations in Experiment 1. Data points show the mean latency across 5 subjects.

Eye movement recording. The subjects' eye movements were monitored with a search coil technique similar to that of Collewijn, van der Mark, and Jansen (1975). The data were logged with a CED 1401 computer, using a sampling rate of $200 \mathrm{~Hz}$ and analyzed off line at a later time. In this analysis, saccades were identified with the use of a velocity criterion, and the latency of the first saccade was recorded. The record also appeared on a monitor display, and if this display indicated any difficulties, the record could be reanalyzed manually or discarded. (Such cases constituted only a small proportion, less than $5 \%$, of the total data throughout Experiments 1-4.)

\section{Results and Discussion}

Figure 1 shows the average saccade latencies in Experiment 1 . The gap condition of $100 \mathrm{msec}$ resulted in the expected decrease in latency, although no further decrease occurred when the gap was increased to $200 \mathrm{msec}$. The upward/downward difference (i.e., the lower visual field effect) occurred at all gap durations and all gaze positions. The results were confirmed with an analysis of variance (ANOVA) in which no interactions were significant, but there was a main effect of gap duration $[F(2,8)=40.10$, $p<.01]$. The upward/downward difference, although consistent across conditions, was only marginally significant $[F(1,4)=3.68, p=.1]$ when tested across subjects. This reflects the influence of 1 anomalous subject who yielded a pattern opposite that normally found, with shorter latencies for downward saccades and moreover with an increase in this effect as gaze was depressed. No explanation is available for this unusual pattern: on other occasions (not reported here), the same subject has produced increased latencies for downward saccades. All the other subjects consistently displayed longer latencies to lower visual field targets.

On the basis of the results of this experiment, it appears that the up/down difference in latency is a robust effect and is not directly influenced either by factors relating to the peripheral musculature or by the differential attentional states occurring in the gap conditions. 


\section{EXPERIMENT 2}

It was possible that increased latency for downward saccades is caused by vergence involvement (the vergence involvement hypothesis), since, as discussed in the introduction, downward saccades are observed to be accompanied by convergence. Two possibilities were therefore contemplated in the design of Experiment 2. First, an increased saccade latency might be found whenever refixation of a target required both a saccadic and a vergence movement. Second, we anticipated a possible interaction between the up/down direction in the visual field and the type of vergence movement. The "natural" combinations (downward to near targets and upward to far targets) occur more often in everyday vision than do the opposite pairings. We hypothesized that this could be reflected in a relative decrease in latency for the natural pairings.

\section{Method}

Subjects. Four laboratory workers (including the two authors) served as the subjects.

Stimuli and Procedure. Saccades were elicited in a straightforward target-tracking task, in which a subject initially fixated on an illuminated central target and then made a saccade to a test target that could be in the same plane, a closer plane, or a more distant plane. The targets at different distances were also in either the upper or the lower visual field. Viewing was binocular.

The targets were red LED light sources with a diameter of $3 \mathrm{~mm}$. Four targets (the horizontal targets) were on the horizontal axis at the same viewing distance $(48 \mathrm{~cm})$ as that of the central fixation target. The horizontal targets were positioned at $2^{\circ}$ and $4^{\circ}$ to both the left and the right of the fixation point. Four targets (the near targets) were positioned at $30 \mathrm{~cm}$ from the fixation point, in a configuration along each of the principal diagonal axes. A further four targets (the far targets) were positioned at a viewing distance of $120 \mathrm{~cm}$, arranged in a similar configuration. The target positions are shown in Figure 2. The near targets were positioned on the corners of a square with sides of $2.10 \mathrm{~cm}$, and the far targets were on the corners of a square with sides of $8.37 \mathrm{~cm}$. As seen from the cyclopean viewpoint shown in Figure 2, each oblique target is displaced $2^{\circ}$ horizontally and vertically. Thus the required average conjugate horizontal movement was $2^{\circ}$ for these targets.
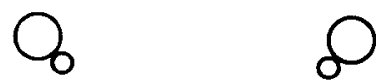

0
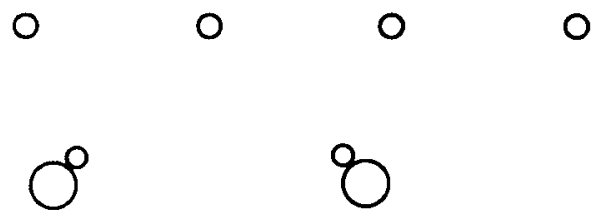

Figure 2. The target configuration of Experiments 2 and 2A as seen from the position midway between the two eyes. The central circle shows the position of the original fixation target. The circles to the left and right of the central circle show the horizontal targets, and the large and small circles that touch show the near and far targets, respectively. For purposes of illustration, the far targets are shown in a slightly more eccentric position than the near targets are.
The initial angle of convergence for a 6-cm interpupillary distance was $7.14^{\circ}$. The convergence angles for the near and far targets were $11.42^{\circ}$ and $2.86^{\circ}$, respectively. Thus, the change in vergence required when fixation changed from the initial position to the near or far targets was $4.28^{\circ}$ in each case $\left(2.14^{\circ}\right.$ angular change in each eye).

Trials were initiated by illuminating the central fixation stimulus. After $1 \mathrm{sec}$, this stimulus was extinguished at the same time as the onset of a peripheral target, and the subject's task was to move the eyes to the peripheral target as rapidly as possible. After a further 1-sec period, the peripheral target was extinguished, the central fixation was illuminated, and the cycle recommenced. A random sequence was programmed with 24 trials in each block, each peripheral target appearing twice. The experiment consisted of 10 blocks, and thus all saccade latencies were based on 20 trials.

Eye movement recording. The horizontal components of the subject's eye movements were monitored with an infrared limbus reflection device (ACS Instruments). The limbus reflection device had a resolution of about 5 arc min and a temporal response higher than $100 \mathrm{~Hz}$. The eye movement data were analyzed as in Experiment 1. The device recorded the horizontal component of eye movements, but it is known that the initiation of the horizontal and vertical components of saccades are very closely synchronized (van Gisbergen, von Opstal, \& Schoenmakers, 1985). We also used a search coil technique in a replication of Experiment 2 with 1 subject; the same pattern of results was obtained.

\section{Results and Discussion}

Figure 3 shows the saccade latencies to the different near and far targets, with the mean latency of saccades to the horizontal targets shown as a dashed line. All saccades to targets in a different depth plane showed increased latencies. In addition, with only one exception, the latencies of saccades to targets in the lower visual field were greater (by an average of $25 \mathrm{msec}$ ) than those to targets in the upper visual field. Detailed statistical analysis will follow presentation of the results from Experiment 3.

For 3 of the subjects, it was possible to measure the latency of the vergence movement in addition to that of the saccade. Movements of divergence commenced at about the time of the saccade and so were not readily measured; the convergence latencies, however, were appreciably shorter (noted also by Yarbus, 1967, and Semmlow \& Wetzel, 1979), and the movements of convergence $\alpha c$ curred well before saccade onset. The mean latencies of the convergence movements were as follows: H.H., near/lower $104 \mathrm{msec}$ and near/upper $100 \mathrm{msec}$; C.M., near/lower $105 \mathrm{msec}$ and near/upper $105 \mathrm{msec}$; C.G., near/lower $110 \mathrm{msec}$ and near/upper $111 \mathrm{msec}$. Thus, there was no substantial difference between the convergence latencies in the lower and upper visual fields; the latency difference appears to be a particular feature of the saccadic system.

\section{Experiment 2A}

In Experiment 2, there were no obliquely positioned targets in the same plane as that of the original fixation point. In order to examine whether the up/down difference would be different if targets were in the same plane, a partial replication of Experiment 2 was carried out, with the authors as subjects. The design of Experiment 2 was 

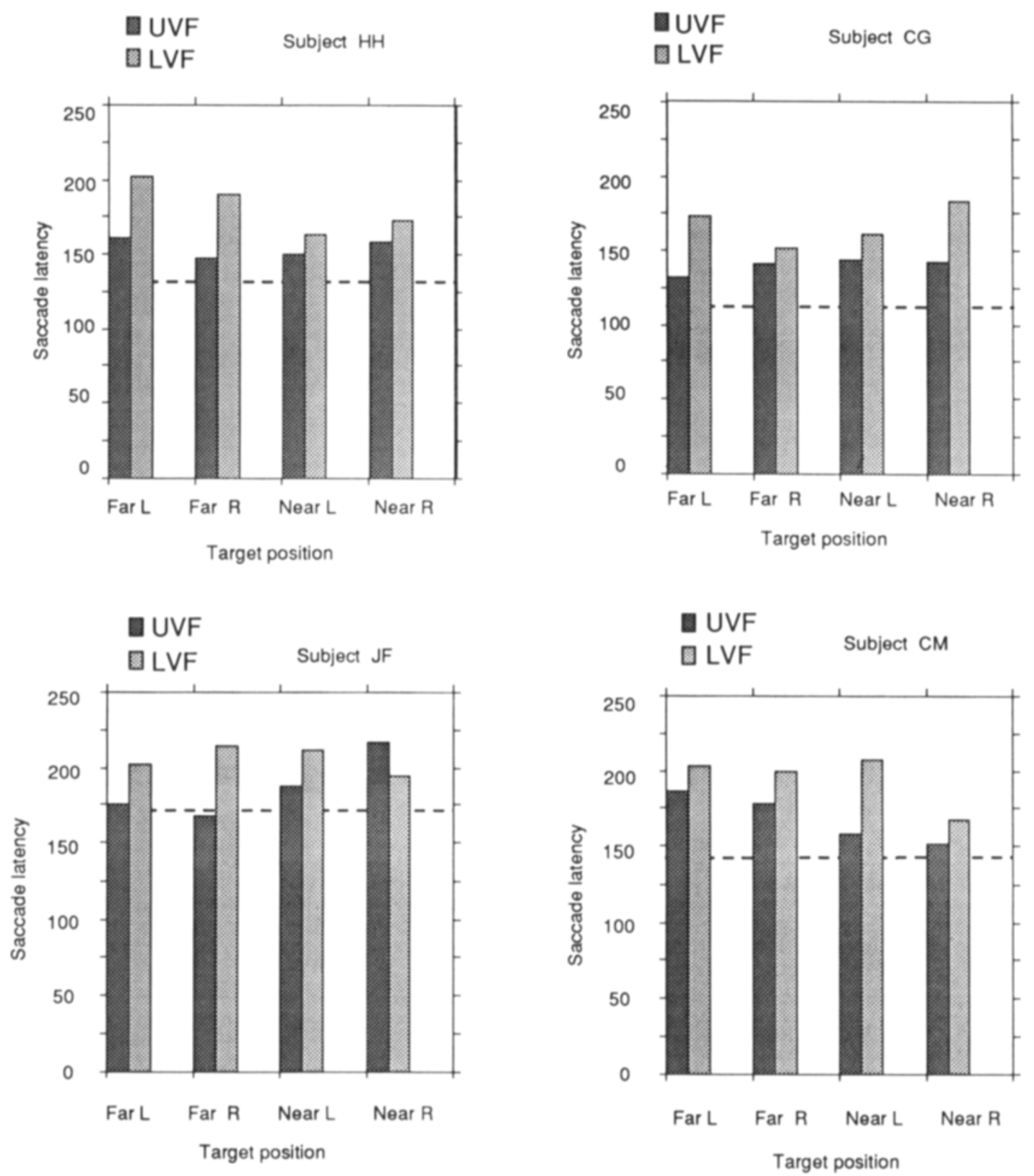

Figure 3. Latencies of saccades in Experiment 2. The plots show, for each subject, the mean saccade latency in milliseconds to targets in the various visual field positions. The dashed lines show the mean latency of the saccades to the horizontally positioned targets in the same plane as that of the fixation points.

followed, with the exception that the near targets were replaced with targets in the same plane as that of the fixation point, spaced around a square with sides of $3.36 \mathrm{~cm}$. These targets required a saccade with horizontal and vertical components of $2^{\circ}$. The results are shown in Figure 4.

The results for targets in the far position were very similar to those of Experiment 2, with an up/down latency difference of $23 \mathrm{msec}$. For targets in the same plane as that of the fixation point, the up/down latency difference was still present, although slightly reduced $(16 \mathrm{msec})$. However, the overall increase of latency in comparison with that for the horizontal targets was no longer obtained.

\section{EXPERIMENT 3}

In Experiment 2, all saccades to targets in a different depth plane showed increased latencies. Such a result does not appear to have been reported previously. These longer latencies may be caused by a link between saccades and vergence movements. It is also possible, however, that the increased latency is a consequence of target disparity. The target configuration shown in Figure 2 is as seen from a hypothetical cyclopean eye. When the actual viewing eyes are considered, the targets also possess disparity. In consequence, the stimulus has a different eccen- 

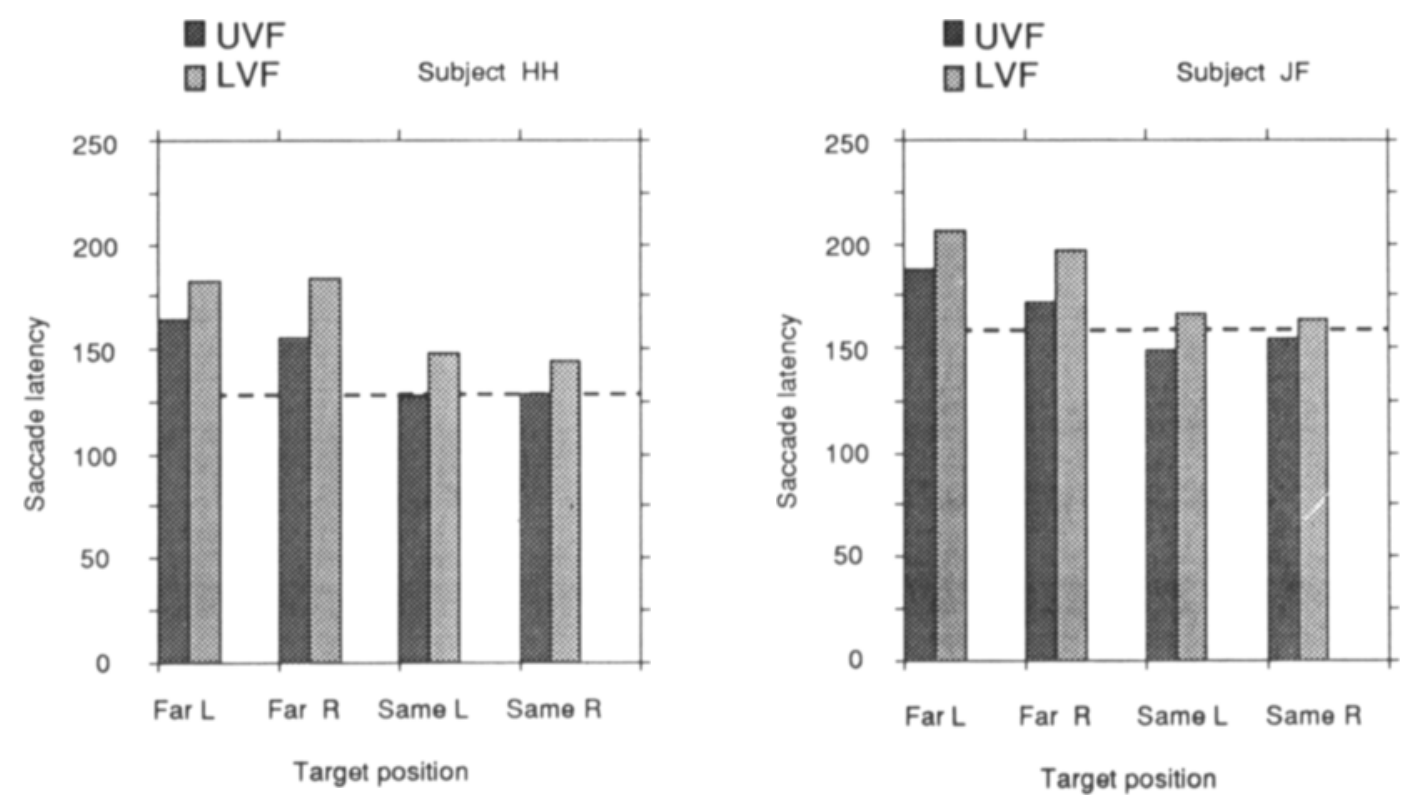

Figure 4. Latencies of saccades in Experiment 2A, in which the obliquely positioned targets could be either in the same plane as, or more distant than, the fixation point. The plots show, for each subject, the mean saccade latency in milliseconds to targets in the various visual field positions. The dashed lines show the mean latency of the saccades to the horizontally positioned targets in the same plane as that of the fixation points.

tricity in each eye. To test whether disparity affected saccade latencies, in Experiment 3 we used the same design as that of Experiment 2, but the subjects viewed the targets monocularly.

\section{Method}

Except for the viewing condition, the method was the same as that of Experiment 2. Each subject was positioned so that the preferred eye was aligned with the central axis of the apparatus and the other eye was occluded. A small vertical displacement was made to the near and far targets to ensure that all were visible from the central position.

\section{Results and Discussion}

The results are shown in Figure 5. Under monocular viewing, the substantial increase in latency for targets in different depth planes is no longer evident.

The results from Experiments 2 and 3 were analyzed together in a three-way ANOVA. The three factors were the viewing mode (binocular/monocular), depth plane (near/far), and visual field (up/down). The monocular/ binocular difference $[F(1.3)=158.2, p<.001]$ and the lower/upper visual field difference $[F(1.3)=64.04, p<$ $.01]$ both presented highly significant effects. The far/near variable had no effect at all, either alone or in interaction. The hypothesis that saccade latencies would be increased when vergence movements were also required was strongly supported. There was no support, however, for the hypothesis that targets occurring in positions consistent with natural ecology (up/far and down/near) would elicit shorter latency saccades than would those occurring in inconsistent positions (up/near and down/far).
There was an interesting interaction between the effects of visual field and viewing mode $[F(1.3)=29.0, p=$ $.01]$. The difference between upper and lower visual fields was reduced from a mean of $25 \mathrm{msec}$ in binocular viewing to a mean of $9 \mathrm{msec}$ in monocular viewing. This suggests, on the one hand, that target disparity and/or vergence movements may contribute to the upward/downward latency difference. On the other hand, however, it should be ncted that the upward/downward latency differences were not completely eliminated under monocular viewing. The up/down difference in latency in the monocular viewing condition was compared statistically in each subject by using the latencies for individual saccades in the two directions (between 100 and 120). For H.H., the difference was $14.2 \mathrm{msec}(t=6.37, p<.01)$; for C.G., $11.3 \mathrm{msec}(t=2.75, p<.01)$; for J.F., $12.8 \mathrm{msec}(t=$ $3.43, p<.01)$; and for C.M., $5.5 \mathrm{msec}(t=1.54$, $p>.1)$.

Under monocular viewing, the overall increase in latencies of saccades to targets in different depth planes was no longer apparent. We expected that under the monocular viewing condition the eyes would show both saccades and vergence movements, as was shown under the binocular viewing condition, since accommodative and other cues to vergence were still present. This was not the case, however. Although both eyes showed saccadic movements, it was rare for these saccades to be accompanied by vergence movements in the viewing eye. In contrast, the occluded eye showed irregular slow drifts, usually starting after the completion of the saccade. Experiment 3 thus differs from Experiment 2 in the absence of both 

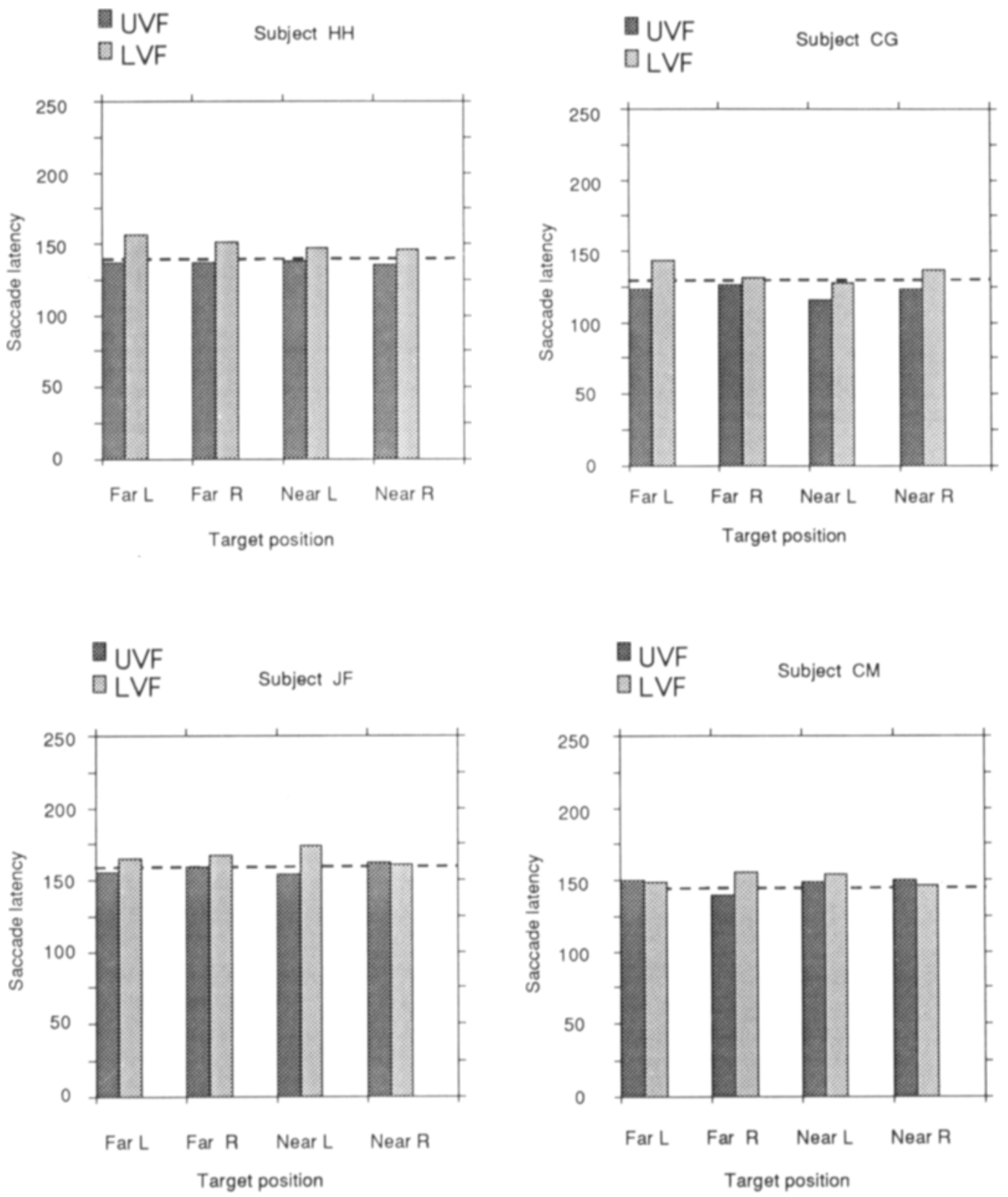

Figure 5. Latencies of saccades in Experiment 3, in which viewing was monocular. The plots show, for each subject, the mean saccade latency in milliseconds to targets in the various visual field positions. The dashed lines show the mean latency of the saccades to the horizontally positioned targets in the same plane as that of the fixation points.

disparity and appropriate vergence movements. We cannot unequivocally conclude whether the latency increase to targets in different depth planes is related to sensory factors or to oculomotor factors.

\section{EXPERIMENT 4}

In all of the experiments to date in which the up/down visual field difference has been demonstrated, the observers have been upright. Consequently, it has not been possible to tell whether the effects relate to the head/body vertical axis or to the visual-environment/gravitation vertical axis. In Experiment 4, we measured saccade latencies from 2 observers who were lying horizontally on a couch, with the axis joining the eyes vertical. Viewing was binocular.

\section{Method}

Two conditions of stimulus arrangement and eye movement recording were used. In Condition $\mathbf{H}$, the arrangement of the stimulus lights was changed so that the lights in the fixation plane were gravitationally vertical, or horizontal on the egocentric axis. The lights were thus in the same physical relationship to the eyes as 
in Experiment 2. In this condition, the horizontal components of the subject's eye movements were monitored. In Condition V, on the other hand, the stimulus lights were arranged as before (lights in the fixation plane horizontal). The lights in the fixation plane were thus aligned in the observer's vertical head plane. A record of vertical eye movement was made by rotating the plane of the eye movement sensor. Although these records of vertical eye movements were inaccurate, it was possible to detect the start of the saccades without difficulty. Two subjects (J.F. and H.H.) participated in both conditions.

\section{Results and Discussion}

In Condition $\mathrm{H}$, there was no significant difference in saccade latency between upward and downward orientations in the gravitational vertical. The mean latencies (egocentric direction in parenthesis) for the fixation plane stimuli were, for J.F., up (left) $162 \mathrm{msec}$ and down (right) $161 \mathrm{msec}$, and for H.H., up (left) $134 \mathrm{msec}$ and down (right) 140 msec. On the other hand, the results of Condition $\mathrm{V}$ indicated that the up/down latency difference is determined by the egocentric vertical. The mean latencies for the fixation plane stimuli (oriented in the egocentric vertical) were, for J.F. up $150 \mathrm{msec}$ and down $164 \mathrm{msec}$, and for H.H., up $147 \mathrm{msec}$ and down $175 \mathrm{msec}$.

Thus Experiment 4 showed convincingly that the latency difference is a feature of the egocentric vertical rather than the gravitational vertical, although this does not establish whether the effect is primarily retinal or oculomotor.

\section{GENERAL DISCUSSION}

We have confirmed that saccade latencies are systematically longer to targets in the lower visual field than to those in the upper visual field. The visual field effect does not depend on the vertical starting position of the eye in the orbit (Experiment 1), and it is related to the visual (egocentric) vertical, rather than the gravitational vertical (Experiment 4). An explanation in terms of peripheral musculature thus appears unlikely. The effect appears to be specific to saccadic eye movements, since it was not found for convergence movements elicited in the course of target acquisition (Experiment 2). In addition, it was shown that although a time gap of $100 \mathrm{msec}$ between the fixation offset and the target onset resulted in a decrease of around $\mathbf{4 0} \mathrm{msec}$ in saccade latency, the size of the decrease was exactly the same for upward and downward movements (Experiment 1). The speedup produced by the gap effect has been attributed to a premotor facilitation of target processing (Reuter-Lorenz, Hughes, \& Fendrich, 1991). The lack of interaction between the gap factor and the up/down difference suggests that the up/down difference is operating independently of this premotor facilitation.

One of the motivations for the present study was to explore the relationship between the up/down difference and the involvement of the vergence system, since there is evidence that downward saccades are accompanied by convergence. In Experiment 2, we found a general increase in saccade latency when the target appeared in a different depth plane. This increase did not occur in monocular viewing (Experiment 3 ). There was no evidence, how- ever, of any interaction between this effect and the up/down difference. In particular, saccade latencies were no shorter to combinations consistent with normal visual ecology (down/near and up/far) than to those that were inconsistent. The results thus do not support any'simple explanation for the up/down difference in terms of vergence involvement.

In all conditions in which targets were presented binocularly in a plane different from the plane of fixation, an increased saccadic latency was, found (Experiments 2 and 2A). This specific finding does not appear to have been reported previously. A control condition (Experiment 2A) confirmed that no latency increase occurred when targets were presented in the plane of fixation but were otherwise exactly identical to those showing the effect. The increase might be related to sensory differences (disparity, accommodative blur), to the requirement to co-program a vergence movement, or to some combination of these factors. In Experiment 3, the same stimulus configuration was used, but the targets were viewed monocularly. In this experiment, no increases occurred in latencies to targets in different depth planes. In the monocular viewing condition, however, vergence eye movements were not made systematically either. Thus it was not possible to be certain whether the latency increase came about because of stimulus factors (disparity) or motor factors (vergence initiation).

The absence of any straightforward explanation of the upward/downward difference invites more complex speculations. Previc (1990) has related the difference to the differential involvement of upper and lower visual fields in extrapersonal (far) and peripersonal (near) visual space, respectively. His assertion, however, that the saccadic system has significantly greater involvement in the former case seems unsupported. Another possible relationship with visual ecology comes from consideration of the course of normal forward locomotion. It will often occur that the eyes remain fixed on an object as it moves lower in the visual field when an observer is moving forward. There will thus, for a moving observer, be a preponderance of downward tracking eye movements and a consequent preponderance of upward refixation saccades. Forward locomotion will result in an overall anisotropy in saccade frequency, with an increased frequency of upward saccades relative to those downward. If saccade latency is affected by the number of saccades previously made in a particular direction as some theories of automaticity might suggest (Logan, 1988), it is possible that the observed latency asymmetry could be a reflection of the pattern of visual ecology.

\section{REFERENCES}

Collewin, H., Erkelens, C. J., \& Stemman, R. M. (1988). Binocular co-ordination of human vertical saccadic eye movements. Joumal of Physiology, 404, 183-197.

Collewijn, H., van der Mark, F., Jansen, T. C. (1975). Precise recordings of human eye movements. Vision Research, 15, 447-450.

ENRIGHT, J. T. (1984). Changes in vergence mediated by saccades. Journal of Physiology, 350, 5-31. 
ENRIGHT, J. T. (1989). Convergence during human vertical saccades: Probable causes and perceptual consequences. Joumal of Physiology, 410, 45-65.

FindLAY, J. M. (1983). Visual information for saccadic eye movements. In A. Hein \& M. Jeannerod (Eds.), Spatially oriented behavior (pp. 281-303). New York: Springer-Verlag.

Findlay, J. M., \& Harris, L. R. (1984). Small saccades to double stepped targets moving in two dimensions. In A. G. Gale \& F. W. Johnson (Eds.), Theoretical and applied aspects of oculomotor research (pp. 71-77). Amsterdam: Elsevier.

FisCher, B., Boch, R., \& RAMSPERGER, E. (1984). Express saccades of the monkey: Effect of daily training on probability of occurrence and reaction time. Experimental Brain Research, 55, 232-242.

FisCHER, B., \& RAMSPERGER, E. (1984). Human express saccades: Extremely short reaction times of goal directed eye movements. Experimental Brain Research, 57, 191-195.

HaCkman, R. (1940). An experimental study of the variability in ocular latency. Journal of Experimental Psychology, 27, 546-558.

Heywood, S., \& Churcher, J. (1980). Structure of the visual array and saccadic latency: Implications for oculomotor control. Quarterly Journal of Experimental Psychology, 32, 335-341.

HowarD, I. P. (1982). Human visual orientation. Chichester, U.K.: Wiley.

LoGAN, G. D. (1988). Towards an instance theory of automatization. Psychological Review, 95, 492-527.

Miles, W. R. (1936). The reaction time of the eye. Psychological Monographs, 47, 268-293.

MiLleR, L. K. (1969). Eye movement latency as a function of age, stimulus uncertainty and position in the visual field. Perceptual Motor Skills, 28, 631-636.
Nakayama, K. (1977). Geometric and physiological aspects of depth perception. In S. Benton (Ed.), Three dimensional imaging (Society of Photo-optical Instrumentation Engineers: Proceedings. Vol. 120, pp. 1-9).

Previc, F. H. (1990). Functional specialization in the lower and upper visual fields in humans: Its ecological origins and neurophysiological implications. Behavioral \& Brain Sciences, 13, 519-575.

Reuter-Lorenz, P. A., Hughes, H. C., \& Fendrich, R. (1991). The reduction of saccadic latency by prior offset of the fixation point: An analysis of the gap effect. Perception \& Psychophysics, 49, 167-175.

SASLOW, M. G. (1967). Effects of components of displacement-step stimuli upon latency for saccadic eye movement. Journal of the Optical Society of America, 57, 1030-1033.

Semmlow, J. L., \& Wetzel, P. (1979). Dynamic contributions to the components of binocular vergence. Journal of the Optical Society of America, 69, 639-645.

Van Gisbergen, J. A. M., van Opstal, A. J., \& Schoenmakers, J. J. M. (1985). Experimental test of two models for the generation of oblique saccades. Experimental Brain Research, 57, 321-336.

WeNBAN-Smith, M. G., \& FindLAY, J. M. (1991). Express saccades: Is there a separate population in humans? Experimental Brain Research, 87, 218-222.

YARBus, A. L. (1967). Eye movements and vision (L. A. Riggs, Trans.). New York: Plenum. (Original work published 1965)

(Manuscript received February 4, 1991; revision accepted for publication February 18, 1992.) 